\title{
In vitro 3D Spheroid Culture Developed on the Parafilm Surface Using HEK- 293 Cells
}

\author{
Erdal EROĞLU* \\ *Manisa Celal Bayar University, Faculty of Engineering, Department of Bioengineering, MANISA, Turkey
}

\begin{abstract}
Preclinical research to predict the effects of drugs and chemicals on humans is commonly carried out either by cell culture studies in vitro condition or on animals in vivo condition. While drug studies tested on cells cultured as a monolayer in plastic flasks are incompatible with realistic results, falsifying findings can also be achieved from in vivo studies performed on different species. In recent years, research on drug tests using spheroid cultures formed by growing cells in three-dimensional (3D) in vitro has attracted great interest. One of the techniques to form 3D spheroids structures is the hanging-drop technique (HDT) in which spheroids are formed by growing the cells in a drop suspended on superhydrophobic surfaces. In this study, HEK-293 cells were investigated on parafilm surfaces displaying superhydrophobic properties by growing in $2 \mu \mathrm{l}$ volume using HDT in terms of spheroid formation. Light microscopy images from spheroid structures were taken on different incubation days and the area of spheroids was measured using the ImageJ program. Our study holds important findings for a chip platform that can be developed for use in vitro drug tests.
\end{abstract}

Key words: spheroids, cell culture, HEK-293 cells, parafilm

\section{Introduction}

In order to assess the safety and efficiency of drugs or chemicals, preclinical studies such as 2 dimensional (2D) in vitro cell culture tests and animal works are unreliable since cells grow as a monolayer on a plastic surface in vitro cell culture condition (Fig. 1A), and completely different species are used in vivo models [1]. Interest in spheroids (Fig. 1B) or organoids obtained from growing cells three dimensional (3D) in vitro has been gradually increasing due to mimicking the complex morphology and physiological tasks of natural tissues [2].

Spheroids were first introduced by Moscona (1952) as cell aggregates in vitro cell suspension [3] and using spheroids in cancer research was recognized by Sutherland in the 1970s. Term of spheroid can simply be described as a spherical (-like) shape cell aggregates grown in vitro

*Corresponding author: Address: Faculty of Engineering, Department of Bioengineering Manisa Celal Bayar University, 45140, Manisa TURKEY. E-mail address: erdal.eroglu@ cbu.edu.tr, Phone: +902362012455 
condition. To form spheroids, there are numerous techniques such as hanging-drop technique, liquid overlay technique, microfluidics devices, and cultures on low-adhesive substrates [2]. The hanging-drop technique (HDT) is one of the widely used technique and which is based on sedimentation of cells growing in a droplet incubated upside down on a sterile petri dish (Fig. 1B) [4].

A

2D cell culture

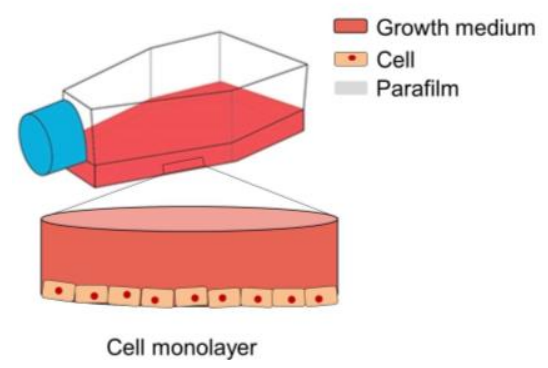

B

3D cell culture

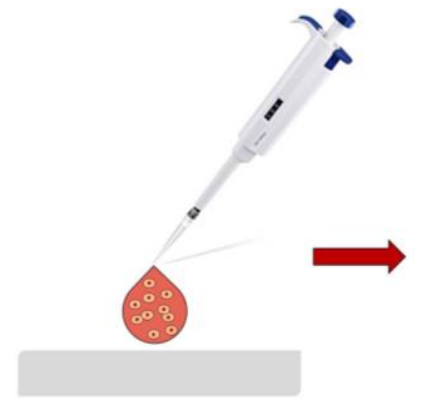

Growth medium

- Trypsinized cell

Parafilm

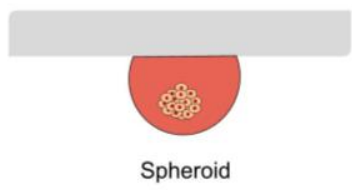

Figure 1. A) $2 \mathrm{D}$ in vitro cell culture and B) $3 \mathrm{D}$ in vitro cell culture

For high-throughput fabrication of spheroids, defined size (a few hundred micrometers) and composition are very crucial parameters in supplying an adequate amount of oxygen to the cells located at the core of spheroids and in preventing necrotic damage [2]. Superhydrophobic surfaces, minimizing contact with a solid substrate, and controlling positional adhesion, is an important prerequisite for a high-throughput spheroid formation at low cost [5].

Parafilm, mainly made of paraffin wax, is highly hydrophobic and resistant to many chemical compounds such as acidic and basic solutions and weak organic solvents. It is a suitable material for microfluidic paper-based analytical devices ( $\mu$ PADs) due to its low cost, hydrophobicity, and sticky and stretchable nature $[6,7]$.

The objective of this study was to form 3D spheroid structures on the Parafilm surface as a platform mimicking natural tissues in order to use in tests analyzing drug efficiency. Here, we present Parafilm as a new spheroid formation platform. Fig. 1B shows a schematic of the spheroid formation in $2 \mu \mathrm{l}$ volume of cell suspension on the Parafilm surface. Our results showed that Parafilm has good physical and chemical properties for spheroid formation with its high hydrophobicity and safety and it would be a promising platform material for microfluidic biochips.

\section{Materials and Method}

\subsection{Maintaince of cell line and culture conditions}

Human epithelial cell line, HEK-293, was kindly provided to us by Dr. Ersin AKINCI at Akdeniz University and cultured in DMEM medium supplemented with $10 \%$ bovine serum (v/v) and $1 \%$ 
antibiotics/antimycotics solution (v/v) (DMEM-10). HEK-293 cells were then maintained at $37^{\circ} \mathrm{C}$ and 5\% CO2 in an incubator (Nuve EC 160). HEK-293 cells grown on plastic flasks as a monolayer were collected by trypsinization and cells in suspension mixed with trypan blue dye (\% 0.2) (1:1 v/v) were counted using a Neubauer counting chamber under a light microscope (Olympus, CKX53). More than $80 \%$ confluent cells with at least $90 \%$ viability were used in the cell culture experiments.

\subsection{Characterization of Parafilm}

In order to determine the thickness of Parafilm, 15 measurements from different areas were taken using an electronic digital caliper.

The surface hydrophobicity of the Parafilm membrane was analyzed measuring the contact angle using a goniometer (Krüss Advance Drop Shape Analyzer, DSA100). The membrane was cut into a thin strip before analyzed. For the measurement of the contact angle, measurements were taken from $2 \mu l$ of distilled water ( $\mathrm{pH} 7$ ) or diiodomethane was dropped on the Parafilm strip.

The geometric mean method which is based on the contact angle measurement of 2 different liquids deposited on to a solid surface, was used to calculate the surface free energy of Parafilm using equation (1) described by Fernandez and Khayet (2015) [8]:

$$
\sqrt[2]{\gamma_{s}^{d} \gamma_{l}^{d}}+\sqrt[2]{\gamma_{s}^{n d} \gamma_{l}^{n d}}=\gamma_{l}(1+\cos Q)
$$

Where $\gamma_{s}^{d}$ is the dispersive component of the solid; $\gamma_{l}^{d}$ is the dispersive component of the liquid; $\gamma_{s}^{n d}$ is the non-dispersive component of the solid; $\gamma_{l}^{n d}$ is the non-dispersive component of the liquid; $\gamma_{l}$ is the total surface free energy or surface tension of the liquid and Q is the contact angle measured between the liquid and the solid under study.

From the total surface tension and surface tension components estimated by the methods described above, the solubility parameter $(\delta)$ of the Parafilm surface was calculated using the equation (2) [8]:

$$
\delta=\left(e_{c}\right)^{1 / 2}
$$

Where $e_{c}\left(\mathrm{~mJ} \mathrm{~m}^{-3}\right)$ is the cohesive energy density, which is related to $\gamma_{s}\left(\mathrm{~mJ} \mathrm{~m}^{-2}\right)$ as equation (3) [8]:

$$
e_{c}=\left(\gamma_{s} / 0.75\right)^{3 / 2}
$$

\subsection{Spheroid formation using HDT}

For spheroid formation using HDT, previously published methods were followed with minor modifications [4, 9]. Parafilm was sealed to the upper part of a sterile Petri dish (90 mm 
diameter; Isolab) using a forceps and Petri dishes, parafilm side up, were sterilized by incubating in a laminar flow hood under UV exposure for $20 \mathrm{~min}$ (Biobase) (Fig. 2A). After UV sterilization, $2 \mu 1$ of cells suspended in culture media (1200 cells/2 $\mu 1$ DMEM-10) was pipetted onto the Parafilm surface to form a drop (Fig. 2B). The bottom part of the Petri dish $(85 \mathrm{~mm}$ diameter; Isolab) was filled up to half with sterile phosphate-buffered saline (PBS) to provide moisture and prevent drops from drying. The lid bearing drops was carefully closed and Petri dishes were incubated upside down at $37^{\circ} \mathrm{C}$ and $5 \% \mathrm{CO}_{2}$ in a humidified incubator.

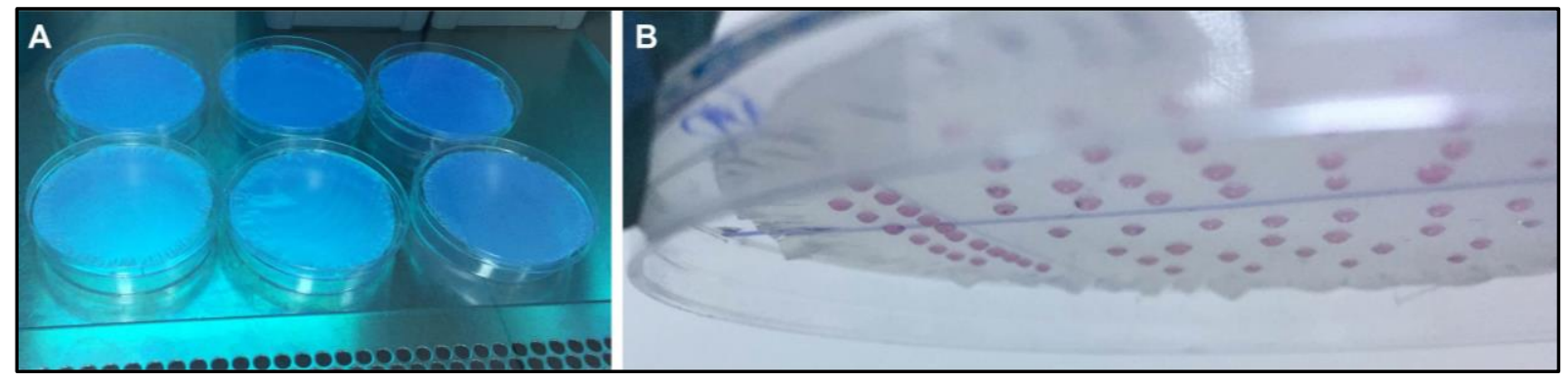

Figure 2. A) UV streilization of Parafilm and B) Drops of cell suspension on the parafilm surface.

\subsection{Light microscopy and morphometric analysis of spheroids}

Spheroid images were acquired every day over 9 days, using an Inverted Light Microscope (Olympus, CKX53) and analyzed using ImageJ Software. The diameter and area of at least 4 different spheroids were measured at different incubation times. Also, spheroids were stained with trypan blue solution (\% 0.2) (1:1 v/v) to observe dead and live cells under the microscope.

\subsection{Statistical test}

Statistical analyses of experimental data were performed using Minitab ${ }^{\circledR} 17$ software. Differences between means of diameters measured on different incubation time were determined using oneway analysis of variance (ANOVA) with a multiple comparison with Tukey's test $(p>0.05)$. The table showing diameters of spheroids were presented as means \pm standard deviation.

\section{Results}

\subsection{Thickness of Parafilm and contact angle measurements}

The average thickness of Parafilm was measured $135 \pm 1 \mu \mathrm{m}$. The contact angle of water and diiodomethane on the Parafilm surface was measured $111.15 \pm 0.55$ and $67.53 \pm 0.41$, respectively (Fig. 3). 


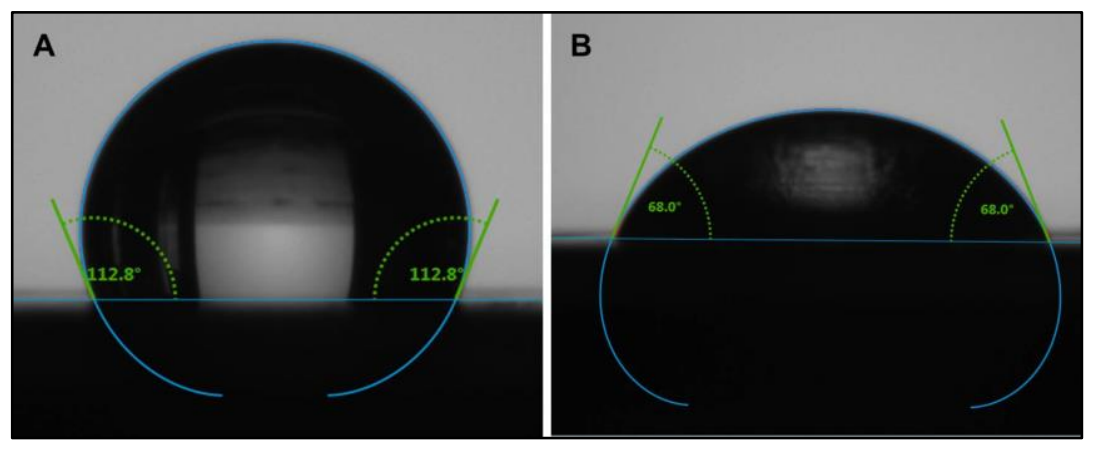

Figure 3. Contact angle measurements of A) water B) diiodomethane on Parafilm surface

The surface free energy of Parafilm was calculated 12.47 using contact angles of water and diiodomethane on the Parafilm. From surface free energy, the solubility parameter was calculated 8.23 for Parafilm. The surfaces with the lower surface energy result in higher contact angle, thus display high hydrophobicity [10].

\subsection{Morphometric analysis of spheroids}

Trypsinized cells suspended in growth medium were observed to start clump together in 2 hours of incubation and full spheroid-like cell aggregates were achieved at the end of the day-1 incubation. The perfect spheroid shape was being observed through 7 days following 2 days of incubation. At the end of the day-14, a lot of dead cells surrounding spheroid were observed (Fig. 4). Irregular spheroid shapes observed in day-1 is getting in a more regular spheroidal shape with incubation time; however, the spherical shapes were seen to be disaggregating with excess incubation due to the dying cells at the surface. In short, more compact spheroids were observed after day-1.

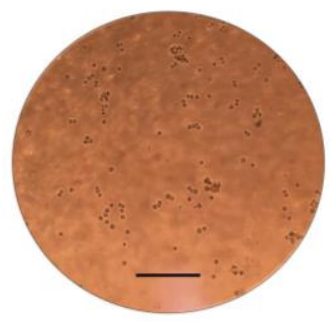

$\mathrm{Oh}$

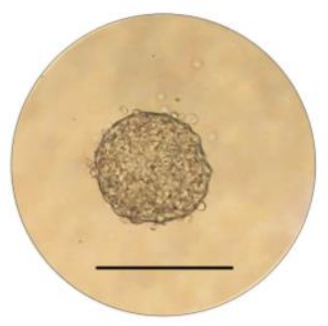

Day-3

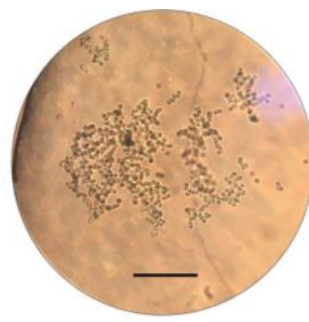

$2 \mathrm{~h}$

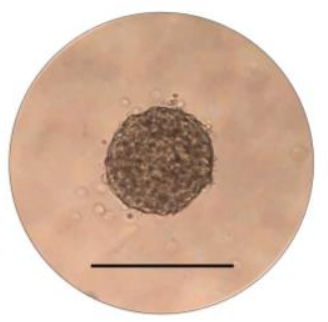

Day-4

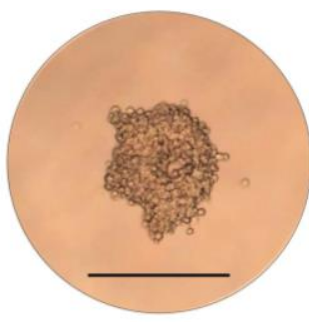

Day-1

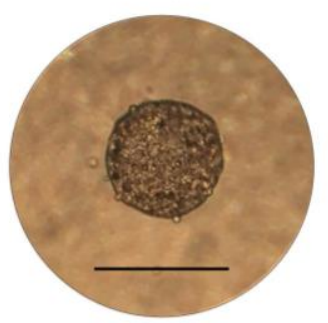

Day-7

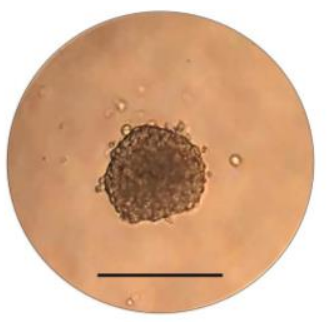

Day-2

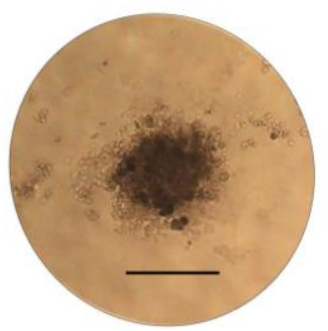

Day-14

Figure 4. Images of spheroids on the parafilm surface observed through 14 days. Scale bars correspond to $250 \mu \mathrm{m}$. 
The highest diameter of spheroids was measured $212.73 \mu \mathrm{m}$ at day-1 using Image $\mathbf{J}$ software. Following incubation days (through day-1 to -7) the spheroid size remained almost the same with approximately $192 \mu \mathrm{m}$. At day-14 the exact size of spheroids could not be measured due to the excess number of dead cells surrounding the spheroid. The areas of spheroids were calculated $35530 \mu \mathrm{m}^{2}$ at day-1 and it remained approximately $29000 \mu \mathrm{m}^{2}$ during day-7 (Table 1). Statistically significant differences in the diameters between the groups were not detected $(\mathrm{p}>0.05)$.

Table 1. Calculated area (with standard deviations) and diameter of spheroids.

\begin{tabular}{ccccccc}
\hline & Day-1 & Day-2 & Day-3 & Day-4 & Day-7 & Day-14 \\
\hline $\begin{array}{c}\text { Area of spheroids } \\
\left(\boldsymbol{\mu m}^{2}\right)\end{array}$ & $35525 \pm 253$ & $29131 \pm 160$ & $28813 \pm 310$ & $29266 \pm 558$ & $28902 \pm 743$ & $\mathrm{Nd}^{*}$ \\
$\begin{array}{c}\text { Diameter of } \\
\text { spheroids }(\boldsymbol{\mu} \mathbf{m})\end{array}$ & 212.73 & 192.64 & 191.58 & 193.08 & 191.88 & $\mathrm{Nd}$ \\
\hline
\end{tabular}

*Nd: Not detected

Also, spheroids were stained with trypan blue dye to differentiate dead and live cells at the surface of spheroids. The number of the dead cell population was increased with incubation time. There were many cells that were imaged in blue color surrounding the spheroid surface at the end of the day-7 and many more cells were observed spread around the spheroid structure at day-14 (Fig. 5).

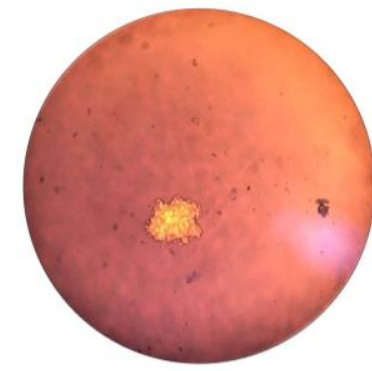

Day-1

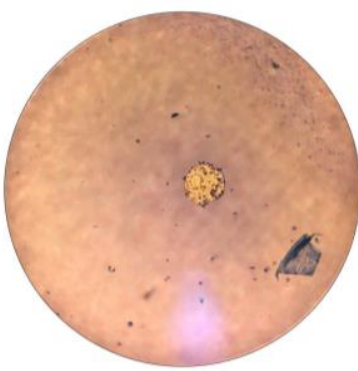

Day-3

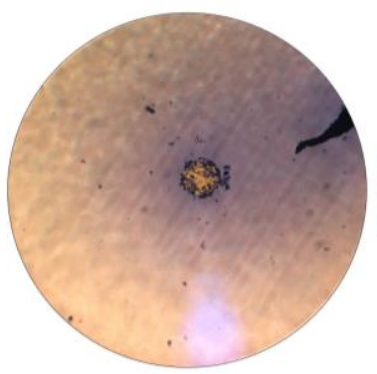

Day-7

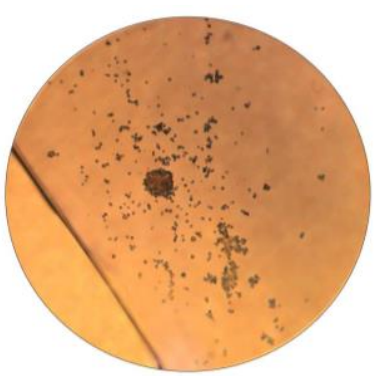

Day-14

Figure 5. Images of spheroids stained with trypan blue dye

\section{Discussion}

In the present work, we were able to observe spheroid structures formed by HEK-293 cells on the Parafilm surface using the HDT technique. Various parameters such as cell type, cell density, microwell or microstructure dimensions, and duration of incubation influence the spheroid diameter. In order to produce spheroids in the desired diameter, cell size is the most crucial 
parameter that should be taken into consideration [11]. In a study performed with HepG2 $\left(10^{7}\right.$ cells $/ \mathrm{ml})$ and COS-7 $\left(10^{5}\right.$ cells $\left./ \mathrm{ml}\right)$ cells, spheroids in $200 \mu \mathrm{m}$ and $80 \mu \mathrm{m}$ size were produced, respectively. The spheroid size of two different cell lines did not display an arithmetical increase with cell number used. This conflict can be explained by the fact that Cos-7 cells have a larger cell diameter than HepG2 cells. [12]. Corroborating with our findings, Liu and co-workers produced spheroids with $201 \mu \mathrm{m}$ in diameter from HEK-293 cells cultivating them in a spinner flask operated at $50 \mathrm{r} / \mathrm{min}$ for 7 days [13]. Unlike this study, however, the method described here requires no specialized equipment or reagents and is therefore highly cost-effective. In another study, spheroids were produced from HEK-293 cells using the HDT technique. In comparison to our work, $10 \mu \mathrm{l}$ cell suspension was directly dropped on a petri dish and 20 times more cells were used in the aforementioned study [14]. With the new method we developed, similar findings using less volume and cell number on the Parafilm surface have been achieved. The decreased cell number was reported leading to the formation of less compact spheroids. However, it was also speculated surface hydrophobicity was found as a key parameter in the production of more compact spheroids (with a smaller diameter) since cells are easily getting concentrated in suspension on hydrophobic surfaces [15]. Considering the calculated surface energy value, it can be seen that the Parafilm was found quite hydrophobic. Besides, HDT was evaluated as a far superior technique compared to round-bottom wells and Matrigel embedding techniques in order to form well-organized spheroids. Taken together, the production of compact spheroids can be attributed to the high hydrophobicity of Parafilm, which leads to ease of gathering of cells in suspension despite using less cell number.

\section{Conclusions}

In summary, the 3D spheroid culture of HEK-293 cells has been successfully produced on the Parafilm surface using HDT. As a result, we have achieved the formation of 3D spheroids that are similar to previous studies, using a reduced volume of cell suspension and cell number on the Parafilm surface. High hydrophobicity of the Parafilm, leading to gathering cells in a drop incubated upside down, helps to the formation of compact spheroids. Therefore the coating biochips with Parafilm-like high hydrophobic materials may increase the efficiency of spheroid formation and so the development of cost-effective and equipment-free drug test systems with higher performance.

\section{Acknowledgements}

This work was partially supported by TÜBİTAK 2209/A university students research project support program. The author would like to thank undergraduate student Fulya ÜNALP for providing a contribution to some experimental settings of this study.

\section{References}

[1] Ivanov DP, Grabowska AM. Spheroid arrays for high-throughput single-cell analysis of 
spatial patterns and biomarker expression in 3D. Sci Rep 2017;7:1-12.

[2] Laschke MW, Menger MD. Life is 3D: Boosting Spheroid Function for Tissue Engineering. Trends Biotechnol 2017;35(2):133-144.

[3] Moscona A, Moscona $\mathrm{H}$. The dissociation and aggregation of cells from organ rudiments of the early chick embryo. J Anat 1952;86(3):287-301.

[4] Metzger W, Sossong D, Bächle A, Pütz N, Wennemuth G, Pohlemann T et al. The liquid overlay technique is the key to formation of co-culture spheroids consisting of primary osteoblasts, fibroblasts and endothelial cells. Cytotherapy 2011;13(8):1000-12.

[5] Neto AI, Correia CR, Oliveira MB, Rial-Hermida MI, Alvarez-Lorenzo C, Reis RL et al.. A novel hanging spherical drop system for the generation of cellular spheroids and high throughput combinatorial drug screening. Biomater Sci 2015;3(4):581-85.

[6] Kim YS, Yang Y, Henry CS. Laminated and infused Parafilm ${ }^{\circledR}$ - paper for paper-based analytical devices. Sensors Actuators, B Chem 2018;255:3654-61.

[7] Mettakoonpitak J, Henry CS. Electrophoretic separations on Parafilm-paper-based analytical devices. Sensors Actuators, B Chem 2018;273:1022-28.

[8] Fernández V, Khayet M. Evaluation of the surface free energy of plant surfaces: toward standardizing the procedure. Front Plant Sci 2015;6:510.

[9] Moraes GS, Wink MR, Klamt F, Silva AO, da Cruz Fernandes M. Simplified Low-Cost Methodology to Establish, Histologically Process and Analyze Three-Dimensional Cancer Cell Spheroid Arrays. Eur J Cell Biol 2020;99(5):151095.

[10] Chieng BW, Ibrahim NA, Ahmad Dau N, Talib ZA. Chapter 8 - Functionalization of Graphene Oxide via Gamma-Ray Irradiation for Hydrophobic Materials. In: Rashid SA, Raja Othman RNI, Hussein, MZ, editors. Synthesis, Technology and Applications of Carbon Nanomaterials, Elsevier; 2019, p. 177-203.

[11] Moshksayan K, Kashaninejad N, Warkiani ME, Lock JG, Moghadas H, Firoozabadi B et al. Spheroids-on-a-chip: Recent advances and design considerations in microfluidic platforms for spheroid formation and culture. Sensors Actuators, B Chem 2018;263:15176.

[12] Patra B, Chen YH, Peng CC, Lin SC, Lee CH, Tung YC. A microfluidic device for uniform-sized cell spheroids formation, culture, harvesting and flow cytometry analysis. Biomicrofluidics 2013;7(5):54114.

[13] Liu H, Liu X-M, Wu B-C, Ye L-L, Ni X-P, Wang Q-W et al. Effects of Hydrodynamics on Aggregates Formation, Growth and Metabolism of HEK 293 Cells in Suspension Culture. Chin J Biotechnol 2006;22(1):101-6.

[14] Foty R. A simple hanging drop cell culture protocol for generation of 3D spheroids. J Vis Exp 2011;(51):2720.

[15] Cavo M, Delle Cave D, D’Amone E, Gigli G, Lonardo E, del Mercato LL. A synergic approach to enhance long-term culture and manipulation of MiaPaCa-2 pancreatic cancer spheroids. Sci Rep 2020;10(1):1-11. 